\title{
Medidas Antropométricas para o Acompanhamento do Estado Nutricional de Crianças e Adolescentes com Câncer, o que utilizar na Prática Clínica?
}

\author{
Anthropometric Measures to Monitor the Nutritional Status of Children with \\ Cancer, which Should be Used in the Practical Clinic? \\ Medidas Antropométricas para el Acompañamiento del Estado Nutricional de \\ Niños y Adolescentes con Cáncer, ¿que Utilizar en la Práctica Clínica?
}

\author{
Andréa Vieira Pereira Coradine' ${ }^{1}$; Mara Albonei Dudeque Pianovski ${ }^{2}$ Estela Iraci Rabito ${ }^{3}$
}

\begin{abstract}
Resumo
Introdução: A avaliação do estado nutricional de crianças e adolescentes com câncer é fundamental para a elaboração do plano de cuidados nutricionais adequado, entretanto as alteraçóes da própria doença e do tratamento podem dificultar essa avaliação. Objetivo: Descrever quais são os métodos antropométricos mais utilizados na avaliação do estado nutricional de crianças e adolescentes com câncer. Método: Realizou-se revisão sistemática da literatura de publicaçôes referentes à avaliação nutricional de crianças e adolescentes com câncer, dos últimos dez anos nas bases de dados MEDLINE, PubMed, Web of Science e LILACS. Resultados: Foram incluídos nove artigos, nos quais foram destacados a amostra, o objetivo e os métodos de avaliação nutricional utilizados. A avaliação de dados isolados da antropometria como peso e estatura de crianças e adolescentes com câncer não é suficiente, uma vez que essa população apresenta alteração da composição corporal. Conclusão: Na prática clínica, a utilização da circunferência do braço, circunferência muscular do braço e dobra cutânea tricipital é indicada para avaliação e acompanhamento da evolução do estado nutricional.
\end{abstract}

Palavras-chave: Avaliação Nutricional; Estado Nutricional; Neoplasias; Criança; Adolescente; Revisão

\footnotetext{
${ }^{1}$ Nutricionista. Mestre em Saúde da Criança e do Adolescente pela Universidade Federal do Paraná (UFPR). Curitiba (PR), Brasil. E-mail: andrea_ufpr@ yahoo.com.br.

${ }^{2}$ Médica-Pediatra Hematologista e Cancerologista. Doutora em Saúde da Criança e do Adolescente pela UFPR. Chefe do Serviço de Oncologia Pediátrica do Hospital de Clínicas da UFPR. Curitiba (PR), Brasil. E-mail: albonei@yahoo.com.br.

${ }^{3}$ Nutricionista. Doutora em Medicina. Clínica Médica pela Universidade de São Paulo (USP). Professora-Adjunta do Curso de Nutrição da UFPR. Curitiba (PR), Brasil. E-mail: estelarabito@yahoo.com.br.

Endereço para correspondência: Andréa Vieira Pereira Coradine. Rua Emílio Cornelsen, 376, apto. 501 - Ahú. Curitiba (PR), Brasil. CEP: $80540-220$.

E-mail: andrea_ufpr@yahoo.com.br.
} 


\section{INTRODUÇÃO}

A avaliação do estado nutricional (EN) de crianças e adolescentes com câncer é necessária para que a intervenção nutricional ocorra de modo a garantir o crescimento e desenvolvimento normal, melhora do sistema imunológico e das condiçôes de resposta ao tratamento antineoplásico e, dessa forma, consequente melhora na qualidade de vida ${ }^{1,2}$. O objetivo da avaliaçáo nutricional é identificar pacientes que estão em risco nutricional e fornecer informaçóes necessárias à elaboração de um plano de cuidado à terapia nutricional adequada ${ }^{3}$. Segundo a Organização Mundial da Saúde (OMS) ${ }^{4}$, deve ser realizada, sequencialmente, e, preferencialmente, com associação de diferentes métodos.

A antropometria é um dos métodos mais usados na prática clínica para avaliação da composição corporal e do EN do indivíduo principalmente na infância e na adolescência, por ser simples, não invasiva e de baixo custo $^{5}$. Compreende avaliação do peso, estatura, dobras cutâneas, circunferências e diâmetros em diferentes compartimentos corporais, além de medidas secundárias como índice de massa corporal (IMC), circunferência muscular do braço (CMB) e área muscular do braço (AMB) que são calculadas por fórmulas que utilizam as medidas citadas inicialmente ${ }^{6}$.

O Consenso Nacional de Nutrição Oncológica Brasileiro ${ }^{7}$ sugere que os métodos antropométricos para avaliação do EN do paciente pediátrico oncológico sejam baseados em: a) peso; b) estatura; c) percentual de perda de peso; d) circunferência do braço (CB); e) CMB; f) dobra cutânea tricipital (DCT) (para crianças > de 2 anos); g) perímetro cefálico e torácico (para crianças < de 2 anos); e nos índices: a) IMC; b) peso para estatura; c) peso para idade; d) estatura para idade.

Como a avaliação antropométrica engloba diversos índices e indicadores, é importante determinar quais são aqueles que melhor identificam o $\mathrm{EN}$ de crianças e adolescentes com câncer para auxiliar o profissional na priorizaçáo do método para o diagnóstico nutricional. Assim, o objetivo desta revisão da literatura é descrever quais são os métodos antropométricos mais utilizados para avaliaçáo do EN de crianças e adolescentes com câncer.

\section{MÉTODO}

Para atingir o objetivo proposto do artigo, foi realizada uma revisão sistemática da literatura com o objetivo de fornecer uma síntese a respeito do tema apresentado, a fim de auxiliar o profissional a decidir qual método antropométrico utilizar na avaliação do EN de crianças e adolescentes com câncer. Para isso, foi realizada uma busca nas bases de dados: MEDLINE, PubMed, LILACS e Web of Science por meio dos seguintes descritores em português e inglês: ("avaliação nutricional" OR "estado nutricional" OR "composição corporal" OR antropometria) AND ("câncer OR neoplasia). Utilizaram-se os limites: humanos, crianças, adolescentes, de 2002 a 2014, em línguas inglesa, portuguesa e espanhola. A busca dos artigos foi realizada no período de junho a setembro de 2014.

O resultado da busca nas bases de dados resultou em 2.154 artigos. Para avaliação da elegibilidade dos artigos, foi realizada análise dos seguintes critérios, sempre obedecendo a essa ordem: avaliação do título; avaliação do resumo; disponibilidade de obter o artigo na íntegra; e avaliação dos resultados que deveriam descrever o EN e/ou composição corporal dos pacientes pelos métodos propostos. Por meio da avaliação do título e resumo, 36 foram elegíveis para esta revisão. Desses, nove foram excluídos após avaliação do resumo, pois avaliaram o EN de jovens adultos sobreviventes de câncer na infância e 18 excluídos por apenas descreverem o EN e não compararem diferentes métodos de avaliação. No final da análise, nove artigos foram incluídos.

Os trabalhos incluídos nesta revisão foram artigos originais com crianças e/ou adolescentes com câncer, publicados em revistas indexadas que tinham como objetivo comparar mais de um método de avaliação do EN, ou que apresentaram na seção de resultados a análise de pelo menos dois métodos de avaliação do EN. Destacaram-se a amostra, a idade, o objetivo do estudo e os métodos de avaliação do EN utilizados.

A principal questáo a ser respondida por meio desta revisão sistemática é quais índices e medidas antropométricos, possíveis de serem aplicados na rotina clínica/ambulatorial, são eficazes para identificar o EN de crianças e adolescentes com câncer. Os artigos incluídos foram avaliados de acordo com a classificaçáo do Oxford Centre for Evidence-Based Medicine $e^{8}$ que avalia o grau de evidência científica dos trabalhos de acordo com o desenho do estudo. Alguns fatores foram limitantes para essa análise como: a) qualidade científica dos artigos analisados, sendo os nove artigos grau de evidência $\mathrm{C}^{8}$ e nenhum trabalho enquadrado como ensaio clínico randomizado. Isso se explica pelo fato de o objetivo dos autores ter sido descrever o EN e/ou correlacioná-lo com algum outro fator como número de complicaçóes; b) amostra não homogênea, com pacientes oncológicos e pacientes com outras doenças não citadas, mas que receberam transplante de medula óssea (TMO).

\section{RESULTADOS E DISCUSSÃO}

Dos nove trabalhos avaliados, um utilizou apenas antropometria ${ }^{9}$ para avaliar o EN dos pacientes e oito, 
além das medidas antropométricas, fizeram uso de algum outro método como exames bioquímicos ${ }^{10}$, impedância bioelétrica (BIA) ${ }^{11}$, absorciometria de feixe duplo (DEXA) $)^{12-14}$, plestimografia ${ }^{15,16}$ e avaliação da massa celular corporal por contagem de potássio corporal ${ }^{17}$. Dos nove artigos incluídos neste trabalho, oito foram encontrados na base de dados PubMed, sendo três também localizados na base MEDLINE ${ }^{10,17}$. Um artigo foi encontrado na base LILACS ${ }^{11}$.

Em relação à utilização da antropometria para avaliação da composição corporal em crianças e adolescentes com câncer, os principais métodos utilizados foram IMC (9 artigos $)^{9-17}$, medidas antropométricas do braço (8 artigos), como DCT ${ }^{10-16}$, dobra cutânea do braço $(\mathrm{DCB})^{12,16}$, $\mathrm{CB}^{9,10,14,17}, \mathrm{CMB}^{10,12}$, área gordurosa do braço $(\mathrm{AGB})^{15,16}$, $\mathrm{AMB}^{11}$. Em duas publicaçôes, foram utilizados os índices peso para idade ${ }^{10}$ e/ou estatura para idade ${ }^{10,11}$ e apenas um fez uso de outras dobras cutâneas: dobra cutânea subescapular (DCSE) ${ }^{16}$ e dobra cutânea suprailíaca $(\text { DCSI })^{16}$. Um artigo utilizou percentagem de peso ideal ${ }^{16}$. A Tabela 1 descreve as características de cada artigo.

Até o momento, não existe um padrão-ouro de avaliaçáo do EN de crianças e adolescentes com câncer, nem valores antropométricos de referência para essa população específica. As neoplasias podem levar o paciente a desenvolver resposta inflamatória à agressão, com liberação de citocinas e hormônios anti-inflamatórios, que geram alterações endócrino-metabólicas e imunológicas ${ }^{2}$, como alteração de carboidratos, proteínas e lipídeos, aumento da lipólise, diminuição da síntese de tecido adiposo, inibição da formação de tecido muscular, estimulação da proteólise e da neoglicogênese ${ }^{18}$ no metabolismo. Toda essa alteração metabólica foi observada por Carraro, Schwartz e Behling ${ }^{11}$ ao avaliarem pacientes em quimioterapia. Os autores correlacionaram o excesso de peso com maior percentual de massa gorda $(M G)$ e menor percentual de massa magra (MM) e água corporal total $($ ACT $)(p<0,001)$ avaliados pela BIA. Esse processo de modificação no metabolismo pode dificultar a avaliação do EN pela antropometria e exames bioquímicos ${ }^{1}$. Tazi et al. ${ }^{10}$ observaram que, no momento do diagnóstico de leucemia linfoide aguda (LLA), a frequência de desnutrição varia dependendo da técnica utilizada: 37\% pelo índice peso para idade, $20 \%$ pelo índice estatura para idade, $33 \%$ pelo IMC, $50 \%$ de acordo com a DCT, $39 \%$ pela CB e 28 pacientes pelos valores de albumina sérica. A mesma conclusão foi obtida por Murphy, White e Davies ${ }^{17}$, ao avaliarem 40 crianças e adolescentes em tratamento antineoplásico. Verificaram que $48 \%$ estavam desnutridos de acordo com a contagem de massa celular corporal, nove pacientes por meio do índice peso para estatura, três pacientes de acordo com o IMC, e nenhum pelos valores de albumina, CB e DCT; e por Sala ${ }^{9}$ que encontrou $28 \%$ dos pacientes desnutridos de acordo com IMC, $18 \%$ moderadamente desnutridos, e $45 \%$ gravemente desnutridos utilizando medidas do braço: $\mathrm{CB}$ e DCT.

Os trabalhos realizados a respeito do assunto têm demonstrado que parâmetros IMC para idade, DCT e CMB apresentam boa associação com o EN, sendo recomendada sua utilizaçáo para estudos epidemiológicos ${ }^{2,5,6,17}$. Em crianças e adolescentes com câncer, as medidas do braço são mais precisas para identificar o EN por serem independentes da raça e não sofrerem influência do peso da massa tumoral ${ }^{19,20}$. A avaliaçáo de peso, de estatura, de DCT, de CB e de albumina tem sido descrita como método de mais fácil utilizaçáo para o acompanhamento do EN em Centro de Tratamento Oncológico e para o diagnóstico nutricional da criança e do adolescente ${ }^{14,15}$.

O IMC ou índice de Quetelet, apesar de ser um instrumento utilizado na rotina de pesquisas de campo para diagnóstico nutricional, deve ter sua interpretação realizada com cautela, uma vez que o resultado é derivado de valores de estatura e peso, sendo que este último não representa os diferentes compartimentos do corpo, podendo super ou subestimar a interpretação clínica ${ }^{6,21}$. White, Davies e Murphy ${ }^{16}$ encontraram correlação positiva entre o $\mathrm{z}$-score de IMC $(\mathrm{r}=0,64$ e $\mathrm{p}<0,00001)$, e DCT, DCB, DCSI e DCSE ( $r=0,76, r=0,8, r=0,72$, $\mathrm{r}=0,71$ e $\mathrm{p}=0,000)$ em crianças com câncer em tratamento antineoplásico; e Murphy, White e Davies ${ }^{17}$, utilizando a contagem de massa celular corporal como método-ouro, encontraram correlação entre essa técnica e IMC ( $\mathrm{r}=0,36$ e $\mathrm{p}=0,02)$. Corroborando os resultados descritos, Barr et al. ${ }^{14}$, ao avaliarem 99 crianças com câncer, correlacionaram IMC $x$ DCT $(r=0,39)$, IMC x CB (r=0,73), IMC x MM obtido por DEXA $(r=0,50)$ e IMC x MG obtido por DEXA $(r=0,60)$ sendo todos resultados significativos $(\mathrm{p}<0,001)$.

Apesar de os estudos ${ }^{15,16}$ apresentarem correlação entre gordura corporal (GC) e peso e percentual de peso ideal, esses devem ser avaliados com atenção por apresentarem limitaçóes semelhantes ao IMC. Durante o tratamento antineoplásico, White, Davies e Murphy ${ }^{16}$, utilizando plestimografia para avaliar GC, encontraram os seguintes resultados: $\mathrm{GC}$ x z-score de peso $(\mathrm{r}=0,58$ e $\mathrm{p}<0,0001)$ e GC x percentual peso ideal $(\mathrm{r}=0,65$ e $\mathrm{p}<0,00001)$. Os mesmos autores, em 2008, correlacionaram GC x percentual de peso ideal $\left(\mathrm{R}^{2}=0,477\right)^{15}$.

Embora as medidas das dobras cutâneas sejam muito utilizadas em estudos epidemiológicos por ser um método barato e não invasivo para predizer a quantidade de GC, algumas limitaçóes devem ser observadas, entre elas: a) 
Tabela 1. Descrição das características dos estudos

\begin{tabular}{|c|c|c|c|c|}
\hline $\begin{array}{l}\text { Referência/ } \\
\text { Local }\end{array}$ & Amostra & Idade & $\begin{array}{l}\text { Método para } \\
\text { avaliar o estado } \\
\text { nutricional }\end{array}$ & Objetivo \\
\hline $\begin{array}{l}\text { Jaime-Pérez } \\
\text { et al., } 2008^{12} \\
\text { México }\end{array}$ & 102 crianças & $\begin{array}{l}\text { Mediana } \\
6 \text { anos }\end{array}$ & $\begin{array}{l}\text { Peso, estatura, DCT, } \\
\text { IMC, DCB, CMB e } \\
\text { DEXA }\end{array}$ & $\begin{array}{l}\text { Descrever o estado nutricional de } \\
\text { crianças com LLA }\end{array}$ \\
\hline $\begin{array}{l}\text { Tazi et al., } \\
2008^{10} \\
\text { Marrocos }\end{array}$ & $\begin{array}{l}100 \text { crianças e } \\
\text { adolescentes ( } 58 \\
\text { câncer hematológico, } \\
38 \text { tumor sólido e } 4 \\
\text { tumor do SNC) }\end{array}$ & $\begin{array}{l}\text { Média } 7 \\
\text { anos }\end{array}$ & $\begin{array}{l}\mathrm{P} / \mathrm{I}, \mathrm{E} / \mathrm{I}, \mathrm{IMC}, \mathrm{DCT} \\
\mathrm{CB}, \mathrm{CMB} \text { e albumina } \\
\text { sérica }\end{array}$ & $\begin{array}{l}\text { Avaliar a prevalência da má } \\
\text { nutrição no momento do } \\
\text { diagnóstico de câncer em crianças }\end{array}$ \\
\hline $\begin{array}{l}\text { White; Davies; } \\
\text { Murphy, } \\
2008^{15} \\
\text { Austrália }\end{array}$ & $\begin{array}{l}23 \text { crianças em } \\
\text { tratamento ( } 13 \\
\text { câncer hematológico, } \\
9 \text { tumor sólido } \\
\text { e } 1 \text { síndrome } \\
\text { hematológica } \\
\text { submetido a TMO) }\end{array}$ & $\begin{array}{l}5 \text { a } 14 \\
\text { anos }\end{array}$ & $\begin{array}{l}\text { Plestimografia, IMC, } \\
\text { DCT, CB e AGB }\end{array}$ & $\begin{array}{l}\text { Verificar a correlação entre o } \\
\text { percentual de gordura corporal } \\
\text { por plestimografia e medidas } \\
\text { antropométricas de crianças em } \\
\text { tratamento oncológico }\end{array}$ \\
\hline $\begin{array}{l}\text { Murphy; } \\
\text { White; Davies, } \\
009^{17} \\
\text { Austrália }\end{array}$ & $\begin{array}{l}40 \text { crianças e } \\
\text { adolescentes ( } 37 \text { em } \\
\text { tratamento oncológico } \\
\text { e } 3 \text { submetidos a } \\
\text { TMO) }\end{array}$ & $\begin{array}{l}5 \text { a } 16 \\
\text { anos }\end{array}$ & $\begin{array}{l}\text { Estatura, peso, IMC, } \\
\text { CB, DCT e massa } \\
\text { celular corporal por } \\
\text { contagem de potássio } \\
\text { corporal }\end{array}$ & $\begin{array}{l}\text { Avaliar se métodos simples } \\
\text { (IMC, CB, DCT) utilizados } \\
\text { para o diagnóstico do estado } \\
\text { nutricional de crianças em } \\
\text { tratamento oncológico são } \\
\text { capazes de identificar má nutrição } \\
\text { determinada pelo método de } \\
\text { massa celular corporal }\end{array}$ \\
\hline $\begin{array}{l}\text { Collins et al., } \\
2010^{13} \\
\text { Canadá }\end{array}$ & $\begin{array}{l}99 \text { crianças e } \\
\text { adolescentes } \\
\text { no momento do } \\
\text { diagnóstico ( } 87 \text { câncer } \\
\text { hematológico, } 2 \\
\text { câncer do SNC e } 10 \\
\text { tumor sólido) }\end{array}$ & $\begin{array}{l}\text { Mediana } \\
86 \text { meses }\end{array}$ & $\begin{array}{l}\text { DEXA, peso, estatura } \\
\text { e IMC }\end{array}$ & $\begin{array}{l}\text { Avaliar a correlação da ingestão } \\
\text { de macronutrientes imediatamente } \\
\text { anterior ao diagnóstico de câncer } \\
\text { com a composição corporal } \\
\text { em crianças e adolescentes no } \\
\text { momento do diagnóstico de câncer }\end{array}$ \\
\hline $\begin{array}{l}\text { Barr et al., } \\
2011^{14} \\
\text { Canadá }\end{array}$ & $\begin{array}{l}99 \text { crianças (tumores } \\
\text { sólidos + leucemia) }\end{array}$ & $\begin{array}{l}\text { Não } \\
\text { descrito }\end{array}$ & $\begin{array}{l}\text { Estatura, peso, IMC, } \\
\text { DCT e CB, MG e } \\
\text { DEXA }\end{array}$ & $\begin{array}{l}\text { Comparar DCT e CB com IMC e } \\
\text { composição corporal por DEXA em } \\
\text { crianças com diagnóstico de câncer }\end{array}$ \\
\hline $\begin{array}{l}\text { White; Davies; } \\
\text { Murphy, } \\
2011^{16} \\
\text { Austrália }\end{array}$ & $\begin{array}{l}42 \text { crianças e } \\
\text { adolescentes } \\
\text { em tratamento } \\
\text { antineoplásico ( } 16 \\
\text { leucemias e } 26 \text { outros } \\
\text { tipos de câncer) }\end{array}$ & $\begin{array}{l}\text { Média de } \\
11,7 \pm \\
3,1 \text { anos }\end{array}$ & $\begin{array}{l}\text { Percentual de } \\
\text { gordura estimado } \\
\text { por plestimografia } \\
\text { e dobras cutâneas } \\
\text { (DCT, DCB, DCSI e } \\
\text { DCSE), IMC, CB, AGB } \\
\text { e \% de peso ideal }\end{array}$ & $\begin{array}{l}\text { Avaliar a correlação entre as } \\
\text { medidas de avaliação nutricional } \\
\text { e percentual de gordura e deduzir } \\
\text { uma fórmula para estimativa de } \\
\text { percentual de gordura corporal em } \\
\text { crianças com câncer }\end{array}$ \\
\hline $\begin{array}{l}\text { Carraro; } \\
\text { Schwartz; } \\
\text { Behling, } \\
2012^{11} \\
\text { Brasil }\end{array}$ & $\begin{array}{l}19 \text { crianças e } \\
\text { adolescentes } \\
\text { (11 tumores } \\
\text { hematológicos e } 8 \\
\text { tumores sólidos) }\end{array}$ & $\begin{array}{l}\text { Média de } \\
10 \pm 3,6 \\
\text { anos }\end{array}$ & $\begin{array}{l}\text { BIA, estatura, peso, } \\
\text { E/I, IMC, AMB, CB e } \\
\text { DCT }\end{array}$ & $\begin{array}{l}\text { Comparar o estado nutricional } \\
\text { obtido por antropometria e } \\
\text { bioimpedância em crianças } \\
\text { e adolescentes submetidos à } \\
\text { quimioterapia }\end{array}$ \\
\hline $\begin{array}{l}\text { Sala et al. } \\
2012^{9} \\
\text { América } \\
\text { Central }\end{array}$ & 1.787 crianças & $\begin{array}{l}\text { Média de } \\
6,8 \text { anos }\end{array}$ & $\begin{array}{l}\text { Estatura, peso, IMC, } \\
\text { CB e DCT }\end{array}$ & $\begin{array}{l}\text { Determinar a prevalência de } \\
\text { má nutrição em crianças e } \\
\text { adolescentes com câncer no } \\
\text { momento do diagnóstico e } \\
\text { correlacionar com desfechos } \\
\text { clínicos }\end{array}$ \\
\hline
\end{tabular}

DCT=dobra cutânea tricipital; $\mathrm{IMC}=$ índice de massa corporal; $\mathrm{DCB}=$ dobra cutânea bicipital; $\mathrm{CMB}=$ circunferência muscular do braço; $\mathrm{DEXA}=\mathrm{absorciometria}$ de raios-X de dupla energia; LLA=leucemia linfoblástica aguda; $\mathrm{P} / \mathrm{E}=$ peso para estatura; $\mathrm{CB}=$ circunferência do braço; $\mathrm{SNC}=$ sistema nervoso central; $\mathrm{P} / \mathrm{I}=$ =peso para idade;

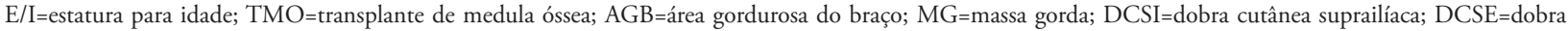
cutânea subescapular; $\mathrm{BIA}=$ impedância bioelétrica; $\mathrm{AMB}=$ área muscular do braço. 
alterações na quantidade e distribuição da GC, de acordo com a idade, sexo, genética, doença, etnia e atividade física; b) variaçóes inter e intra-avaliadores na execução da medição; c) precisão dos instrumentos de coleta; d) inexistência de padróes de referências locais ${ }^{22,23,24}$. White, Davies e Murphy ${ }^{15}$ encontraram correlação positiva entre a percentagem de GC avaliada por plestimografia por deslocamento de ar com $\mathrm{CB}\left(\mathrm{R}^{2}=0,363\right)$ e $\mathrm{AGB}$ $\left(\mathrm{R}^{2}=0,524\right)$ em crianças em tratamento oncológico.

A DCT, medida bastante utilizada por ser de fácil localização e apresentar forte correlação com o percentual total de GC, é recomendada pela $\mathrm{OMS}^{4}$ como índice isolado ou associado na avaliação de crianças, adolescentes, adultos e idosos. No trabalho realizado por Carraro, Schwartz e Behling ${ }^{11}$, os autores observaram correlação positiva $(r=0,74)$ entre DCT e GC obtida por BIA e correlação negativa $(r=0,76)$ entre DCT e MM também obtida por BIA. Para White, Davies e Murphy15, a DCT e percentagem de peso ideal devem ser incorporadas à rotina de avaliação nutricional, por apresentarem correlação com a percentagem de $\mathrm{GC}\left(\mathrm{R}^{2}=0,532\right.$ e $\mathrm{R}^{2}=0,477$, respectivamente) em crianças oncológicas bem nutridas. Avaliando pacientes em tratamento antineoplásico, Murphy, White e Davies ${ }^{17}$ encontraram correlação entre GC por contagem de massa celular corporal e DCT $(r=0,37$ e $\mathrm{p}=0,03)$. Entretanto, a correlação de $\mathrm{MG}$ por DEXA e DCT avaliada por Barr et al. ${ }^{14}$ não foi significativa $(\mathrm{r}=0,25)$.

Outro método para avaliar o EN é estimar os estoques de proteína por meio de medidas de massa muscular. Collins et al. ${ }^{13}$ correlacionaram o consumo de macronutrientes com a composição corporal por DEXA e obtiveram correlação significativa, mas moderada, entre massa corporal magra e percentagem de consumo médio de energia e proteína $(\mathrm{r}=0,33$ e $\mathrm{p}<0,0009)$. Já na prática clínica, essa avaliaçáo pode ser realizada por meio da $\mathrm{CB}$ juntamente com a DCT que são utilizadas na fórmula que avalia a massa muscular do braço, denominada CMB, que considera a reserva de tecido muscular, podendo ser relacionada à massa muscular corporal total ${ }^{6,21}$.

Mesmo que as medidas antropométricas sejam fáceis de serem obtidas, é importante ressaltar que esses valores devem ser interpretados com cautela, pois nem sempre são reais, uma vez que podem ser confundidos por alteraçóes na água corporal, ocasionadas por desidratação, edema, ascite e pela própria alteração hidroeletrolítica que o câncer pode ocasionar. Para Carraro, Schwartz e Behling ${ }^{11}$, a utilização da BIA ao invés de medidas antropométricas nessa populaçáo parece ser melhor, uma vez que a reprodutibilidade é mais fácil e os resultados refletem valores do compartimento corporal, MG, MM e ACT, o que direciona o acompanhamento nutricional.
A má nutrição em pacientes pediátricos interfere na qualidade de vida, prognóstico e sobrevida, sendo necessário o acompanhamento do EN para intervenção precoce quando detectada alguma alteração, permitindo o suporte nutricional adequado nesses pacientes para promover a manutenção do peso, o crescimento linear e melhor tolerância ao tratamento. Os métodos considerados ouro são difíceis de serem reproduzidos na clínica, entretanto os seus resultados são mais precisos do que medidas antropométricas e exames laboratoriais. Jaime-Pérez et al. ${ }^{12}$, ao avaliarem o EN de 102 pacientes pelo IMC, encontraram 12 pacientes desnutridos, 66 eutróficos e 24 com sobrepeso ou obesidade; e, ao realizarem DEXA em 98 desses pacientes, constataram que 16 estavam com valores de GC abaixo do recomendado, 43 adequados e 19 com valores acima do recomendado quando comparados com crianças e adolescentes saudáveis.

Para Murphy, White e Davies ${ }^{17}$, métodos simples de avaliação do EN, como IMC, DCT, perda de peso e os índices peso para estatura, não são suficientes para o diagnóstico nutricional de crianças com câncer, sendo necessária a avaliação de novos métodos mais detalhados para essa população.

Atualmente, especial atenção tem sido dada ao desenvolvimento de métodos de diagnóstico do EN por meio da avaliaçấo celular que pode monitorar alteraçôes da composição corporal e alteraçóes funcionais da membrana celular $^{25}$, importantes para o paciente hospitalizado ou em tratamento agressivo.

A contagem de potássio corporal, a plestimografia e o DEXA são técnicas conhecidas e validadas no meio científico para avaliação da massa celular corporal, entretanto a reprodutibilidade na prática clínica é inviável pela complexidade e alto custo das técnicas ${ }^{26}$. Nesse sentido, por meio dos princípios de funcionamento da BIA, pesquisas apontam que é possível avaliar a integridade da membrana celular por via do valor de ângulo de fase, técnica esta que pode ser reproduzida em ambiente ambulatorial ${ }^{25,27,28}$, podendo se tornar um novo método de avaliação do EN para essa população.

\section{CONCLUSÃO}

Para o diagnóstico do EN de crianças e adolescentes com câncer, a avaliação apenas do peso e estatura não são suficientes, uma vez que essa população apresenta alteração da composição corporal. Na prática clínica, a utilização da CB, CMB e DCT é mais indicada para avaliação e acompanhamento da evolução do EN. Entretanto, a interpretação dos resultados antropométricos não deve ser o único parâmetro para o desenvolvimento do suporte nutricional do paciente; e sim apenas mais um método 
que, somado a outras avaliaçóes (alimentar, clínica, funcional e bioquímica), permite o diagnóstico do EN.

\section{CONTRIBUIÇÕES}

Andréa Vieira Pereira Coradine participou da concepção e planejamento do projeto de pesquisa; obtenção, análise e interpretaçáo dos dados, redação e revisão crítica. Mara Albonei Dudeque Pianovski e Estela Iraci Rabito participaram da obtenção, análise e interpretação dos dados, redação e revisáo crítica.

\section{Declaraçáo de Conflito de Interesses: Nada a Declarar.}

\section{REFERÊNCIAS}

1. Drisko JA, Chapman J, Hunter VJ. The use of antioxidant therapies during chemotherapy. Gynecol Oncol. 2003 Mar;88(3):434-9.

2. Garófolo A, Lopez FA, Petrilli AS. Hight prevalence of malnutrition among patients with solid nonhematological tumors as found by using skinfold and circumference measurements. Sao Paulo Med J. 2005 Nov 3;123(6):277-81.

3. ASPEN Board of Directors and the Clinical Guidelines Task Force. Guidelines for the use of parenteral and enteral nutrition in adult and pediatric patients. JPEN J Parenter Enteral Nutr. 2002 Jan-Feb;26(1 Suppl):1SA138SA.

4. World Health Organization. Physical status: the use and interpretation of anthropometry: report of a WHO Expert Committee. Geneva: WHO; 1995. Who technical report series, 854 .

5. Hoffman DJ, Heymsfield SB, Waitzberg DN. Composição Corpórea. In: Waitzberg DL. Nutrição oral, enteral e parenteral na prática clínica. São Paulo: Atheneu; 2001. p. 225-39.

6. Sant'anna MSL, Priore SE, Franceschini SCC. Métodos de avaliação da composição corporal em crianças. Rev Paul Pediatr. 2009;27(3):315-21.

7. Instituto Nacional de Câncer (BR). Consenso nacional de nutrição oncológica: volume II. Rio de Janeiro: Inca; 2011. p. 25-33.

8. Centre for Evidence-based Medicine. Oxford Centre for Evidence-based Medicine - Levels of Evidence (March 2009) [Internet]. Oxford: CEBM; 2009 [acesso em 2015 ago 28]. Disponível em: http://www.cebm.net/ oxford-centre-evidence-based-medicine-levels-evidencemarch-2009/.

9. Sala A, Rossi E, Antillon F, Molina AL, Maselli T, Bonilla $\mathrm{M}$, et al. Nutritional status at diagnosis is related to clinical outcomes in children and adolescents with cancer: A perspective from Central America. Eur J Cancer. 2012 Jan;48(2):243-52.
10. Tazi I, Hidane Z, Zafad S, Harif M, Benchekroun S, Ribeiro R. Nutritional status at diagnosis of children with malignancies in Casablanca. Pediatr Blood Cancer. 2008 Oct;51(4):495-8.

11. Carraro JL, Schwartz R, Behling EB. Comparação do estado nutricional obtido através de antropometria e bioimpedância em crianças e adolescentes submetidos a tratamento quimioterápico em um hospital no Sul do Brasil. Clin biomed res. 2012;32(1):35-41.

12. Jaime-Pérez JC, González-Llano O, Herrera-Garza JL, Gutiérrez-Aguirre H, Vázquez-Garza E, GómezAlmaguer D. Assessment of nutritional status in children with acute lymphoblastic leukemia in Northern México: A 5-year experience. Pediatr Blood Cancer. 2008 Feb;50(2 Suppl):506-8.; discussion 517.

13. Collins L1, Nayiager T, Doring N, Kennedy C, Webber C, Halton J, et al. Nutritional status at diagnosis in children with cancer I. An assessment by dietary recallcompared with body mass index and body composition measured by dual energy X-ray absorptiometry. J Pediatr Hematol Oncol. 2010 Nov;32(8):e299-303.

14. Barr R, Collins L, Nayiager T, Doring N, Kennedy C, Halton J, et al. Nutritional status at diagnosis in children with câncer. 2. An assessment by arm anthropometry. J Pediatr Hematol Oncol. 2011 Apr;33(3):e101-4.

15. White M, Davies P, Murphy A. Validation of percent body fat indicators in pediatric oncology nutrition assessment. J Pediatr Hematol Oncol. 2008 Feb;30(2):124-9.

16. White M, Davies P, Murphy A. Correlation between nutrition assessment data and percent body fat via plethysmography in pediatric oncology patients. JPEN J Parenter Enteral Nutr. 2011 Nov;35(6):715-22 .

17. Murphy AJ, White M, Davies PSW. The validity of simple methods to detect poor nutritional status in paediatric oncology patients. Br J Nutr. 2009 May;101(9):1388-92.

18. Cravo ML, Glória LM, Claro I. Metabolic responses to tumour disease and progression: tumor-host interaction. Clin Nutr. 2000 Dec;19(6):459-65.

19. Sala A, Pencharz P, Barr RD. Children, cancer and nutrition--A dynamic triangle in review. Cancer. 2004 Feb 15;100(4):677-87.

20. Frisancho AR. New norms of upper limb fat and muscle areas for assessment of nutritional status. Am J Clin Nutr. 1981 Nov;34(11):2540-5.

21. Sigulem DM, Devincenzi MU, Lessa AC. Diagnóstico do estado nutricional da criança e do adolescente. J Pediatr. 2000;76(Supl 3):275-84.

22. Sichieri R, Fonseca VM, Lopes CS. Como medir a confiabilidade de dobras cutâneas. Rev Bras Epidemiol. 1999;2(1-2):82-9.

23. Heyward VH, Stolarczyk LM. Avaliação da composição corporal aplicada. Barueri: Manole; 2000.

24. Petroski EL. Antropometria: técnicas e padronizações. Porto Alegre: Palloti; 1999. 
25. Barbosa-Silva MC, Barros AJD, Wang J, Heymsfield SB, Pierson Jr RN. Bioelectrical impedance analysis: population reference values for phase angle by age and sex. Am J Clin Nutr. 2005 Jul;82(1):49-52.

26. Pichard C, Kyle UG. Body composition measurements during wasting diseases. Curr Opin Clin Nutr Metab Care. 1998 Jul;1(4):357-61.

27. Nagano M, Suita S, Yamanouchi T. The validity of bioelectrical impedance phase angle for nutritional assessment in children. J Pediatr Surg. 2000 Jul;35(7):1035-9.

28. Schwenk A, Beisenherz A, Römer K, Kremer G, Salzberger B, Elia M. Phase angle for bioelectrical impedance analysis remains an independent predictive marker in HIV infected patients in the era of highly active antiretroviral treatment. Am J Clin Nutr. 2000 Aug;72(2):496-501. 


\begin{abstract}
Introduction: The evaluation of nutritional status of children and adolescents with cancer is fundamental to the elaboration of adequate planning for nutritional monitoring, however alterations caused by the disease and/or the treatment may hamper this evaluation. Objective: Describing anthropometric methods mostly used at the evaluation of nutritional status of children and adolescents with cancer. Method: Systematic review of the literature of publications produced within the last ten years referring to nutritional evaluation of children and teenagers with cancer took place based on data from MEDLINE, PubMed, Web of Science and LILACS. Results: Nine articles were studied from which samples were highlighted, objectives and methods for nutritional evaluation were also used. The evaluation of isolated anthropometric data such as weight and height of children and teenagers with cancer is not sufficient once this specific population presents alteration on body composition. Conclusion: The utilization of arm muscle circumference, arm muscle area and triceps skinfold thickness measurements are proposed in order to evaluate and monitor nutritional status in practical clinics.
\end{abstract}

Key words: Nutrition Assessment; Nutritional Status; Neoplasms; Child; Adolescent; Review

\title{
Resumen
}

Introducción: La evaluación del estado nutricional de niños y adolescentes con cáncer es fundamental para la elaboración de un plan de cuidados nutricionales adecuados, sin embargo las alteraciones de la propia enfermedad y del tratamiento pueden dificultar esta evaluación. Objetivo: Describir cuales son los métodos antropométricos más utilizados en la evaluación del estado nutricional de niños y adolescentes con cáncer. Método: Se realizó revisión sistemática de la literatura y de publicaciones referentes a la evaluación nutricional de niños y adolescentes con cáncer, de los últimos diez años en las bases de datos MEDLINE, PubMed, Web of Science y LILACS. Resultados: Se incluyeron nueve artículos, en los cuales fueron destacados la muestra, objetivo y métodos de evaluación nutricional utilizados. La evaluación de datos aislados de la antropometría como peso y estatura de niños y adolescentes con cáncer no es suficiente, una vez que esta población presenta alteración de la composición corporal. Conclusión: En la práctica clínica, la utilización de la circunferencia del brazo, circunferencia muscular y dobla cutánea tricípite es indicada para evaluación y acompañamiento de la evolución del estado nutricional.

Palabras clave: Evaluación Nutricional; Estado Nutricional; Neoplasias; Niño; Adolescente; Revisión 